\title{
The importance of claudin-7 palmitoylation on membrane subdomain localization and metastasis-promoting activities
}

\author{
Sarah Heiler, Wei Mu, Margot Zöller ${ }^{*}$ and Florian Thuma*
}

\begin{abstract}
Background: Claudin-7 (cld7), a tight junction (TJ) component, is also found basolaterally and in the cytoplasm. Basolaterally located cld7 is enriched in glycolipid-enriched membrane domains (GEM), where it associates with EpCAM (EpC). The conditions driving cld7 out of TJ into GEM, which is associated with a striking change in function, were not defined. Thus, we asked whether cld7 serines or palmitoylation affect cld7 location and protein, particularly EpCAM, associations.

Results: HEK cells were transfected with EpCAM and wild type cld7 or cld7, where serine phopsphorylation or the palmitoylation sites (AA184, AA186) (cld $\left.7^{\mathrm{mPalm}}\right)$ were mutated. Exchange of individual serine phosphorylation sites did not significantly affect the GEM localization and the EpCAM association. Instead, cld $7^{\text {mPalm }}$ was poorly recruited into GEM. This has consequences on migration and invasiveness as palmitoylated cld7 facilitates integrin and EpCAM recruitment, associates with cytoskeletal linker proteins and cooperates with MMP14, CD147 and TACE, which support motility, matrix degradation and EpCAM cleavage. On the other hand, only $\mathrm{cld} 7^{\mathrm{mPalm}}$ associates with TJ proteins.
\end{abstract}

Conclusion: Cld7 palmitoylation prohibits TJ integration and fosters GEM recruitment. Via associated molecules, palmitoylated cld7 supports motility and invasion.

Keywords: EpCAM, claudin7, Palmitoylation, Cleavage, Membrane microdomains

\section{Introduction}

Claudins (cld), a family of closely related four-pass molecules, are essential components of tight junctions (TJ) in the apical region of epithelial cells. TJ, which include JAM and occludins, regulate paracellular permeability and epithelial cell polarity [1-4]. As tumor cell dissemination requires loss of cell-cell adhesion, it was expected that claudins be downregulated in cancer. This however was not consistently observed [3,5-7]. Fittingly, claudins, most frequently cld7, also are recovered on the lateral or basal surface [6,8-10]. However, functional activities of claudins out of TJ are poorly defined [5].

Claudins have two palmitoylation sites towards the inner membrane site of the second and forth transmembrane domain $[11,12]$. As described for G-protein coupled

\footnotetext{
* Correspondence: m.zoeller@uni-hd.de; Florian.Thuma@uni-heidelberg.de Department of Tumor Cell Biology, University Hospital of Surgery, Im Neuenheimer Feld 365, 69120 Heidelberg, Germany
}

receptors and tetraspanins [13-15], palmitoylation promotes the recruitment into glycolipid-enriched membrane microdomains (GEM) [16,17], which gather signal transduction $[16,18,19]$ and cytoskeleton anchoring molecules [20]. Palmitoylation also promotes internalization and degradation or integration into multivesicular bodies and exosome delivery [21-24]. Claudins also have several phosphorylation sites $[25,26]$. Cld phosphorylation can prohibit integration into TJ [27], but distinct claudins may respond differently $[28,29]$.

Claudins interact between themselves, additional TJ components and TJ-independent membrane molecules. CD9 and CD81 associate with cld1, the latter being essential for hepatitis $C$ virus infection [30-32]. The interaction of EphrinB1 and EphrinA2 with cld4 affects barrier functions and increases leakiness [33,34]. We described that cld7 directly associated with EpCAM $(\mathrm{EpC})$ in the transmembrane region [35]. EpC-cld7 colocalization is seen in colon, pancreatic and anaplastic 
thyroid carcinoma [36,37]. Pursuing in a rat pancreatic adenocarcinoma the impact of cld7 on EpC activity and vice versa revealed that cld7 recruits EpC into GEM, where both molecules associate with the tetraspanin Tspan 8 and additional transmembrane molecules. These latter associations likely are not based on direct proteinprotein interactions $[36,37]$. A cld $7^{\mathrm{kd}}$ and an $E p C^{\mathrm{kd}}$ revealed a striking reduction in metastasis formation, where cld7 supports tumorigenic features of $\mathrm{EpC}$ by provoking $\mathrm{EpC}$ cleavage and thereby the cotranscription factor activity of its intracellular domain (EpIC) $[38,39]$. $\mathrm{Cld} 7$ also associates with $\mathrm{EpC}$ in hepatocyte progenitors [40]. In congenital tufting enteropathy, characterized by a deletion of $\mathrm{EpC}$ exon 4, the loss of co-localization with cld7 is lethal [41]. Notably, an $\mathrm{EpC}^{\mathrm{ko}}$ is associated with intestine destruction-promoted death within one week after birth, due to the missing association of $\mathrm{EpC}$ with cld7, which is accompanied by reduced cld7 expression [42]. The pathology resembles that of $\operatorname{cld} 7^{\mathrm{ko}}$ mice, which also die within 1 week after birth, due to altered barrier functions and inflammation-promoted gut destruction. The authors discuss the importance of a missing association with integrins and a striking up-regulation of MMP3 [43].

These findings strengthening our hypothesis on TJindependent functions and cooperations of cld7 under (patho)physiological conditions [44-47], we aimed to elaborate the molecular mechanism prohibiting cld7 integration into TJ. As a model we choose HEK cells, which express cld7 at a very low level. HEK cells were transfected with serine- and palmitoylation-site-mutated cld7. We focused on cld7 palmitoylation, as palmitoylation is of critical importance for GEM localization, complex formation and recruitment of cytoskeletal linker and cytosolic signaling molecules [48-51]. To control for activities that depend on the cooperation with EpC, HEK cells were concomitantly transfected with $\mathrm{EpC} \mathrm{cDNA}$ or a point mutated EpC cDNA that does not associate with cld7. Cld7 palmitoylation prohibits integration into the $\mathrm{TJ}$ protein complex. Instead, palmitoylated cld7 is preferentially recruited into GEM, where it associates with integrins and cytoskeletal linker proteins, which promote motility. Motility is additionally strengthened by cleavage of palmitoylated cld7-associated $\mathrm{EpC}$, the co-transcription factor EpIC (EpC intracellular domain) supporting mesenchymal gene expression. Finally, palmitoylated cld7 associates with proteases, which strongly affects invasiveness.

\section{Results}

The activity of cld7 outside of $\mathrm{TJ}$ is still disputed. We described recovery of cld7 in GEM, where it associates with $\mathrm{EpC}$, tetraspanins and integrins, which supports motility and possibly the epithelial mesenchymal transition (36,52). The molecular basis for driving cld7 out of TJ into GEM as well as the GEM-locationdependent associations remained to be explored. We approached these questions by transfecting HEK cells with cld7, where serine phosphorylation sites $(\mathrm{mS})$ and the palmitoylation site at the C-terminal cytoplasmic tail (mPalm) were mutated. The impact particularly on associating EpC was controlled by co-transfecting HEK cells with EpC, exchanging AA279 and AA282 by isoleucin (mAG), which prohibits cld7 binding $(35,36)$.

\section{Claudin7 palmitoylation affects membrane subdomain localization and the association with EpC}

We first evaluated the impact of serine phosphorylation on cld7 recruitment into GEM and on the association with EpC. HEK-EpC cells were transfected with seven different serine phosphorylation site point-mutated cld7 constructs (Additional file 1). WB showed comparable cld7 expression levels in non-mutated/mutated cld7 transfected HEK-EpC cells (Additional file 2a). The exchange of cld7 serines did not affect co-immunoprecipitation of EpC with cld7 (Additional file 2b). WB of sucrose density gradient fractions revealed partial recovery of cld7 in GEM. In the absence of cld7, EpC is mostly recovered in heavy fractions, but becomes partially recruited into light fractions in the presence of cld7. The distribution of $\mathrm{EpC}$ in light sucrose density fractions corresponded to the distribution of cld7. Recovery of EpC in GEM fractions varied between $55 \%-72 \%$ and of cld7 between 57\%-73\%. Thus, single serine mutations did not strongly affect or abolish GEM localization of cld7 and the recruitment of EpC into GEM (Additional file 2c). $\mathrm{Co}$-immunoprecipitation confirmed that anti-EpC precipitates cld7 and anti-cld7 precipitates EpC. Irrespective of cld7 serine mutations, $60 \%-70 \%$ of EpC-cld7 complexes are recovered in GEM. This is demonstrated for $\operatorname{cld} 7^{\mathrm{mS3} 3}, \operatorname{cld} 7^{\mathrm{mS69}}, \operatorname{cld} 7^{\mathrm{mS} 87}$ and $\operatorname{cld} 7^{\mathrm{mS} 204}$ (Additional file 2d), and also accounted for $\operatorname{cld} 7^{\mathrm{mS} 172}$, $\operatorname{cld} 7^{\mathrm{mS} 206}$ and $\operatorname{cld} 7^{\mathrm{mS} 207}$ (data not shown).

We concluded that cld7 is preferentially located in GEM and recruits EpC into GEM. Single serine phosphorylation of cld7 does not considerably contribute to the EpC-cld7 association or to the membrane distribution of cld7 and the EpC-cld7 complex.

We proceeded evaluating the impact of cld7 palmitoylation, a modification known to support protein anchoring in GEM $[16,17]$. HEK-EpC cells were transfected with cld7 carrying a mutation of the palmitoylation site at the C-terminal tail (HEK-EpC-cld $7^{\mathrm{mPalm}}$ ). To control, in addition, whether only cld7-associated $\mathrm{EpC}$ becomes recruited into GEM, HEK-cld7 cells were transfected with EpC cDNA mutated at AA279 and AA282 (HEK$\mathrm{EpC}^{\mathrm{mAG}}$-cld7), which account for the EpC-cld7 association [36]. Flow cytometry and WB confirmed comparable 
$\mathrm{EpC} / \mathrm{EpC} \mathrm{mAG}^{\mathrm{mA}}$ and cld7 / cld $7^{\mathrm{mPalm}}$ expression in transfected HEK cells (Figure 1a).

The efficacy of inhibiting cld7 palmitoylation by the exchange of cysteine AA184 and AA186 was controlled by immunoprecipitation and acyl-biotin exchange in HEK-EpC-cld7 and HEK-EpC-cld $7^{\text {mPalm }}$ lysates in the absence or presence of the palmitoylation inhibitor 2-BP. No palmitoylation signal was detected in the presence of
2-BP, but a strong signal was seen in HEK-EpC-cld7 lysates. A very faint band remained in HEK-EpC$\mathrm{cld} 7^{\mathrm{mPalm}}$. Stripping the gel and blotting with anti-cld7 confirmed equal loading. From there we conclude that mutating AA184 and AA186 sufficed to prevent palmitoylation and also that the C-terminal palmitoylation site mostly accounts for cld7 palmitoylation (Figure 1b). We next evaluated the impact of mutating the cld7

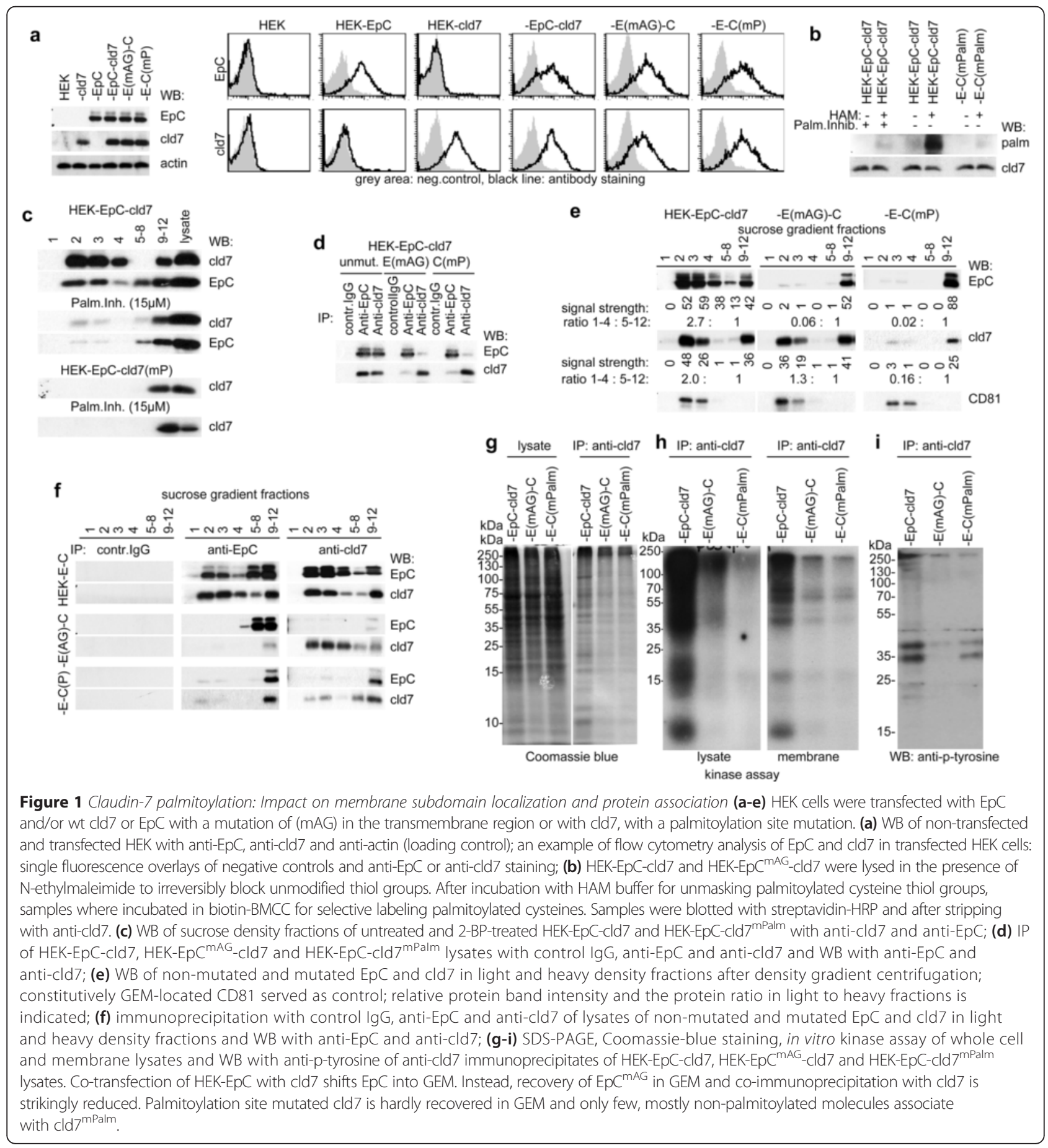


palmitoylation site on the recruitment into GEM and the association with $\mathrm{EpC}$ using the palmitoylation inhibitor (2-BP) [53]. WB of 2-BP-treated HEK-EpC-cld7 cells showed a striking redistribution of cld7 with $>90 \%$ recovery in the heavy fraction. The same accounted for EpC. On the contrary, cld7 was not recovered in GEM and 2BP treatment had no impact on the cld7 GEM distribution in HEK-EpC-cld $7^{\mathrm{mPalm}}$ (Figure 1c). Thus, a considerable part of cld7 is palmitoylated and palmitoylation accounts for GEM recruitment. The finding also strengthens our interpretation that cld7 recruits EpC towards GEM. We proceeded with HEK-EpC-cld $7^{\mathrm{mPalm}}$ and HEK$\mathrm{EpC}^{\mathrm{mAG}}$-cld7 cells, where selectively cld7 palmitoylation and the cld7-EpC association should be distorted.

Co-immunoprecipitation of $\mathrm{EpC}$ and cld7 was strikingly reduced in HEK-EpC ${ }^{\mathrm{mAG}}$-cld7 and HEK-EpC-cld $7^{\mathrm{mPalm}}$ lysates (Figure 1d). In HEK-EpC ${ }^{\mathrm{mAG}}$, $\mathrm{EpC}$ was recovered in heavy sucrose gradient fractions, but cld7 remained enriched in GEM. Instead, cld $7^{\mathrm{mPalm}}$ partly shifted towards the dense fractions. Enrichment of the constitutively GEM-located tetraspanin CD81 in fraction 2-5 was independent of cld7 palmitoylation (Figure 1e). Furthermore, $\mathrm{EpC}$ was not detected in light and was poorly recovered in heavy density fractions in anti-cld7 immunoprecipitates of HEK-EpC-cld $7^{\mathrm{mPalm}}$ lysates. In HEK-EpC ${ }^{\text {mAG }}$-cld7 lysates, anti-EpC weakly coimunoprecipitated cld7 only in heavy density fractions, but cld7 was still recovered in light density fractions of anti-cld7 precipitates (Figure 1f). Furthermore, $\mathrm{cld} 7^{\mathrm{mPalm}}$ and $\mathrm{EpC}^{\mathrm{mAG}}$ severely influenced the phosphorylation status of associated molecules. First to note, SDS-PAGE and Coomassie blue staining of anti-cld7 precipitates revealed a reduction of co-immunoprecipitating molecules in $\mathrm{EpC}^{\mathrm{mAG}}$ and more pronounced $\mathrm{cld} 7^{\mathrm{mPalm}}$ lysates, indicating that $\mathrm{EpC}$ - and cld7-associating molecules are partly overlapping (Figure 1g). Notably, a kinase assay revealed phosphorylation of a considerable number of molecules co-immunoprecipitating with cld7 in HEKEpC-cld7, but not in HEK-EpC ${ }^{\mathrm{mAG}}$-cld7 or HEK-EpC$\mathrm{cld} 7^{\mathrm{mPalm}}$ lysates. The finding was confirmed in a kinase assay with lysates of the membrane fraction. In addition, recovery of $\sim 15 \mathrm{kDa}$ and $\sim 11 \mathrm{kDa}$ phosphorylated proteins, which abundantly immunoprecipitated in whole cell lysates, was poor in the membrane fraction IP. This could be indicative for loosely attached cytosolic proteins (Figure $1 \mathrm{~h}$ ). These molecules and a $\sim 60 \mathrm{kDa}$ protein are exclusively serine or threonine phosphorylated as they were not recovered in an anti-p-tyrosine blot (Figure 1i). We conclude that mostly serine/threonine phosphorylated proteins associated exclusively with palmitoylated cld7.

MALDI-TOF analysis of lower molecular weight proteins of HEK-EpC-cld7, HEK-EpC ${ }^{\mathrm{mAG}}$-cld7 and HEK-EpC-cld $7^{\mathrm{mPalm}}$ lysates, which co-immunoprecipitated with cld7, confirmed that a considerable number of proteins co-immunoprecipitated with cld7 only in HEK-EpC-cld7, but not in HEK-EpC ${ }^{\mathrm{mAG}^{-}}$-cld7 and, more pronounced in HEK-EpC-cld7 ${ }^{\mathrm{mPalm}}$ lysates. Due to very mild lysis condition, many co-immunoprecipitating molecules may be loosely attached and be part of larger protein complexes. Notably, the majority of molecules coimmunoprecipitating with cld7 in HEK-EpC-cld7, HEK$\mathrm{EpC}^{\mathrm{mAG}}$-cld7 and HEK-EpC-cld $7^{\mathrm{mPalm}}$ lysates is engaged in metabolism and ion transport. Instead, an unexpectedly high number of molecules co-immunoprecipitating with cld7 only in HEK-EpC-cld7 lysates is engaged in vesicle transport (Additional file 3).

Briefly, (i) cld7 palmitoylation is essential for stabilization in GEM and for the association with $\mathrm{EpC}$ and other transmembrane and membrane-associated molecules; (ii) the association with palmitoylation-competent cld7 severely affects the activation state of co-immunoprecipitating molecules; (iii) molecules associated with vesicle traffic preferentially associated with palmitoylation-competent cld7, which strengthens our hypothesis of distinct functions of palmitoylated versus non-palmitoylated cld7. The selectivity of palmitoylated cld7 membrane subdomain localization and protein associations offered the possibility to control for palmitoylation-dependent cld7 activities.

\section{Cld7 palmitoylation interferes with cell-cell adhesion}

Formation of $\mathrm{TJ}$ is one of the central activities of clds [54]. As cld7 is also recovered outside of $\mathrm{TJ}$ and palmitoylation strongly affects cld7 location $[35,54,55]$, we first asked, whether palmitoylation directly affects cld7 integration into TJ.

HEK cells express cld 3 at a high, ZO- 1 and cld 4 at an intermediate and cld7 at a very low level (Figure 2a). Cld7 poorly colocalizes with cld3 or ZO-1. However, there is a tendency towards more pronounced colocalization of palmitoylation-deficient cld7 with cld3 and ZO-1. Distinct to cld7, cld4 readily colocalizes with ZO-1 (Figure 2b). Co-immunoprecipitation confirmed that only cld $7^{\mathrm{mPalm}}$, but not palmitoylation-competent cld7 associates with cld3, cld4 and ZO-1. Instead, anti-ZO-1 precipitates cld 3 and cld4, but only cld $7^{\text {mPalm }}$ (Figure 2c). Thus, only non-palmitoylated / palmitoylation-deficient cld7 may contribute to TJ formation.

Similar to cld7, EpC is a homophilic cell-cell adhesion molecule [56]. To control for a potential impact of the association with cld7, CFSE-labeled HEK-EpC-cld7, HEK-EpC ${ }^{\text {mAG }}$-cld7 and HEK-EpC-cld7 ${ }^{\text {mPalm }}$ cells were seeded on a monolayer of HEK-EpC-cld7, HEK-EpC ${ }^{\text {mAG }_{-}}$ cld7 or HEK-EpC-cld7 $7^{\text {malm }}$ cells. Slightly more HEK$\mathrm{EpC}^{\mathrm{mAG}}$-cld7 and HEK-EpC-cld7 ${ }^{\mathrm{mPalm}}$ than HEK-EpC-cld7 cells adhered to the HEK-EpC-cld7 monolayer (Figure 3a). Increased cell-cell adhesion of HEK-EpC ${ }^{\mathrm{mAG}}$-cld7 was due to EpC binding, as it was inhibited by anti-EpC (Figure 3b). This finding supports the interpretation that GEM-located 


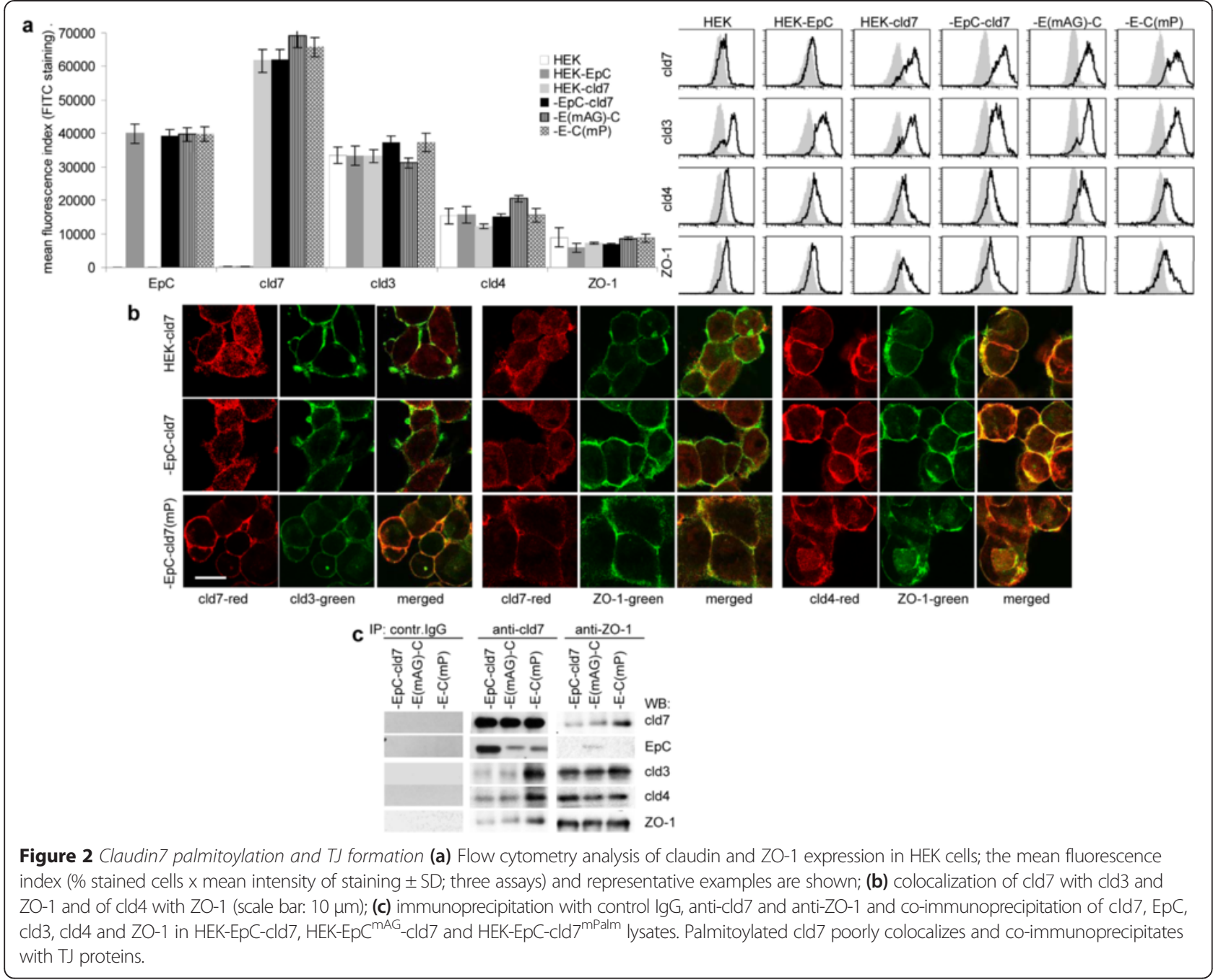

cld7 also interferes with homophilic EpC tetramer cell-cell adhesion.

Cld7 additionally could hamper EpC-mediated cell-cell adhesion by supporting TACE-dependent EpC cleavage [39]. In fact, the TACE inhibitor TAPI strengthened cellcell adhesion (Figure 3b). TAPI abolished EpEx generation and EpEx recovery was reduced in the supernatant of HEK-EpC ${ }^{\mathrm{mAG}}$-cld7 and HEK-EpC-cld7 $7^{\text {mPalm }}$ cells (Figure 3c). Confocal microscopy showed proximity of TACE to EpC only in HEK-EpC-cld7 cells, but very weak colocalization in HEK-EpC ${ }^{\text {mAG}}$-cld7 and HEKEpC-cld $7^{\mathrm{mPalm}}$ cells. Cld7 also colocalized with TACE in HEK-EpC ${ }^{\mathrm{mAG}}$-cld7, but poorly in HEK-EpCcld $7^{\text {mPalm }}$ cells (Figure $3 \mathrm{~d}$ ). WB confirmed partial recovery of TACE in GEM of HEK-EpC-cld7 and HEK$\mathrm{EpC}^{\mathrm{mAG}}$-cld7, but not HEK-EpC-cld7 ${ }^{\mathrm{mPalm}}$ cells (Figure 3e). Finally, anti-TACE co-immunoprecipitates EpC and cld7 in light sucrose density gradient fractions of HEK-EpC-cld7, but not HEK-EpC cld7 $7^{\text {mPalm }}$ lysates (Figure 3f).
Taken together, cld7 palmitoylation prohibits engagement in TJ and interferes with EpC homophilic cell-cell adhesion. Cld7-associated TACE, which supports EpC cleavage, additionally affected EpCAM cell-cell adhesion.

\section{Claudin-7 palmitoylation promotes cell motility}

Based on the finding that palmitoylation hampers cellcell adhesion, we speculated that it might foster motility. The impact of cld7 palmitoylation on motility was evaluated in an in vitro wound healing assay, by transwell migration and by videomicroscopy. Wound closure after scratching a cell monolayer is accelerated in HEK-cld7 and HEK-EpC-cld7 compared to HEK and HEK-EpC cells. Motility of HEK-EpC ${ }^{\text {mAG }}$-cld7 is comparable to that of HEK-EpC-cld7, but motility of HEK-EpC$\operatorname{cld} 7^{\mathrm{mPalm}}$ is reduced (Figure 4a). Transwell migration (Figure 4b) and videomicroscopy (Figure 4c) confirmed that HEK-cld7 and HEK-EpC-cld7 cells migrate significantly faster than HEK and HEK-EpC cells and that migration of HEK-EpC-cld $7^{\mathrm{mPalm}}$ is reduced. These 


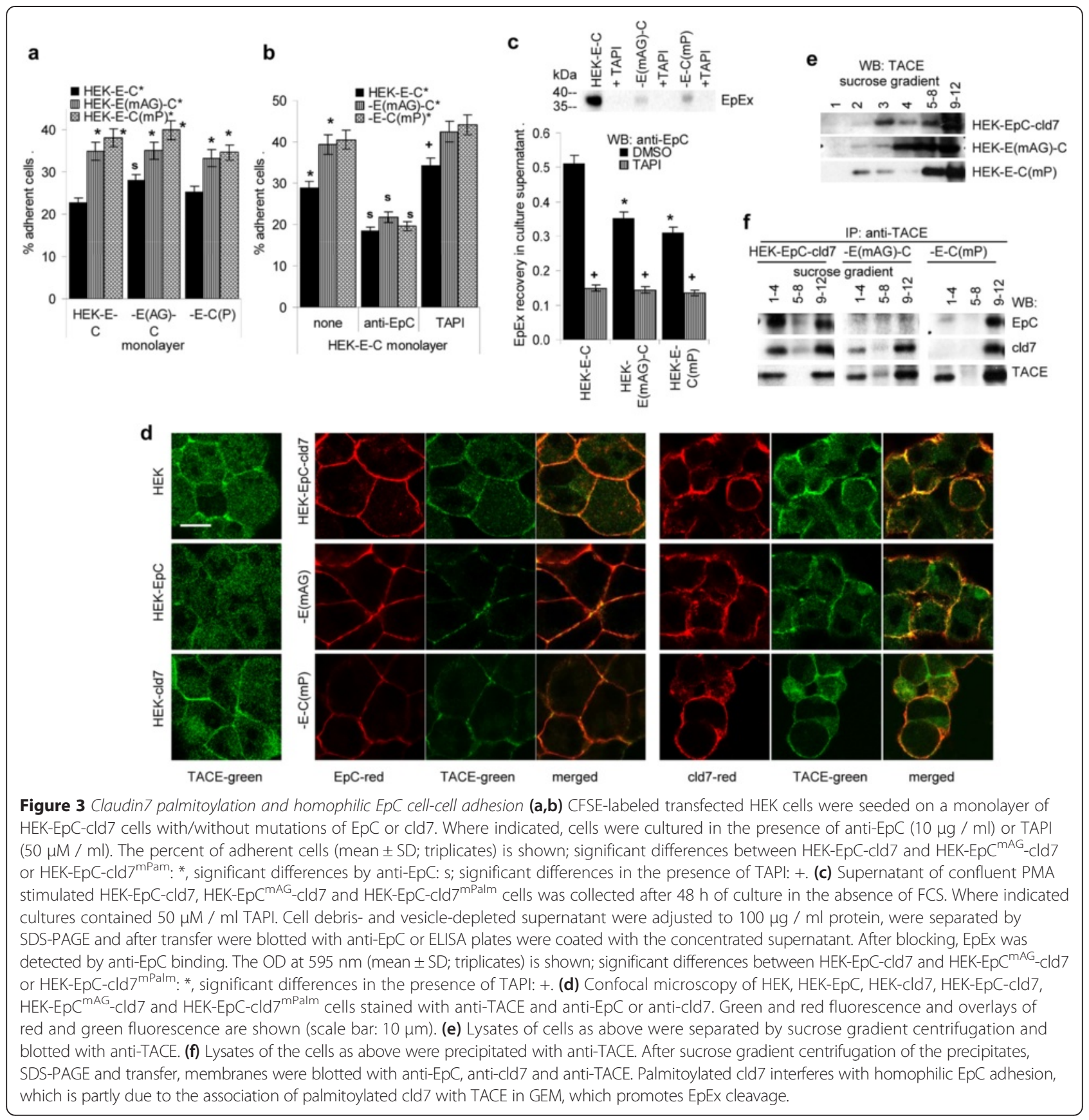

findings pointed towards palmitoylated cld7 actively promoting motility, but also pointed towards a contribution of EpC. The latter suggestion derives from the observation that HEK-EpC ${ }^{\mathrm{mAG}}$-cld7 cells migrate slower than HEK-EpC-cld7 cells.

Phalloidin staining of HEK cells uncovered a disorganized cytoskeleton [57], but a striking enrichment in barbs and strong submembrane bundles in HEK-cld7 and HEK-EpC-cld7 cells. Actin is less efficiently organized in HEK-EpC ${ }^{\text {mAG }}$-cld7 and HEK-EpC-cld7 ${ }^{\text {mPalm }}$ cells (Figure 5a).
HEK cells express the $\alpha 5 \beta 1$ and very weakly the $\alpha 6 \beta 4$ integrin; they highly express CD166. Expression remains unchanged in cld7 transfected cells. Furthermore, (p)-ezrin, (p)-src, (p)-FAK and paxillin expression is not significantly reduced in HEK-EpC ${ }^{\mathrm{mAG}}$-cld7 and HEK-EpC-cld7 ${ }^{\mathrm{mPalm}}$ cells (Figure 5b, 5c). Despite unaltered expression, colocalization of cld7 with integrins, but not with CD166, is weakened in HEK-EpC-cld7 ${ }^{\text {mPalm }}$ and HEK-EpC ${ }^{\text {mAG }}$-cld7 (Figure 5d) and colocalization with ezrin and src is weakened in HEK-EpC-cld $7^{\mathrm{mPalm}}$ cells (Figure 6a). These findings suggested that the cld7-initiated organization of the 


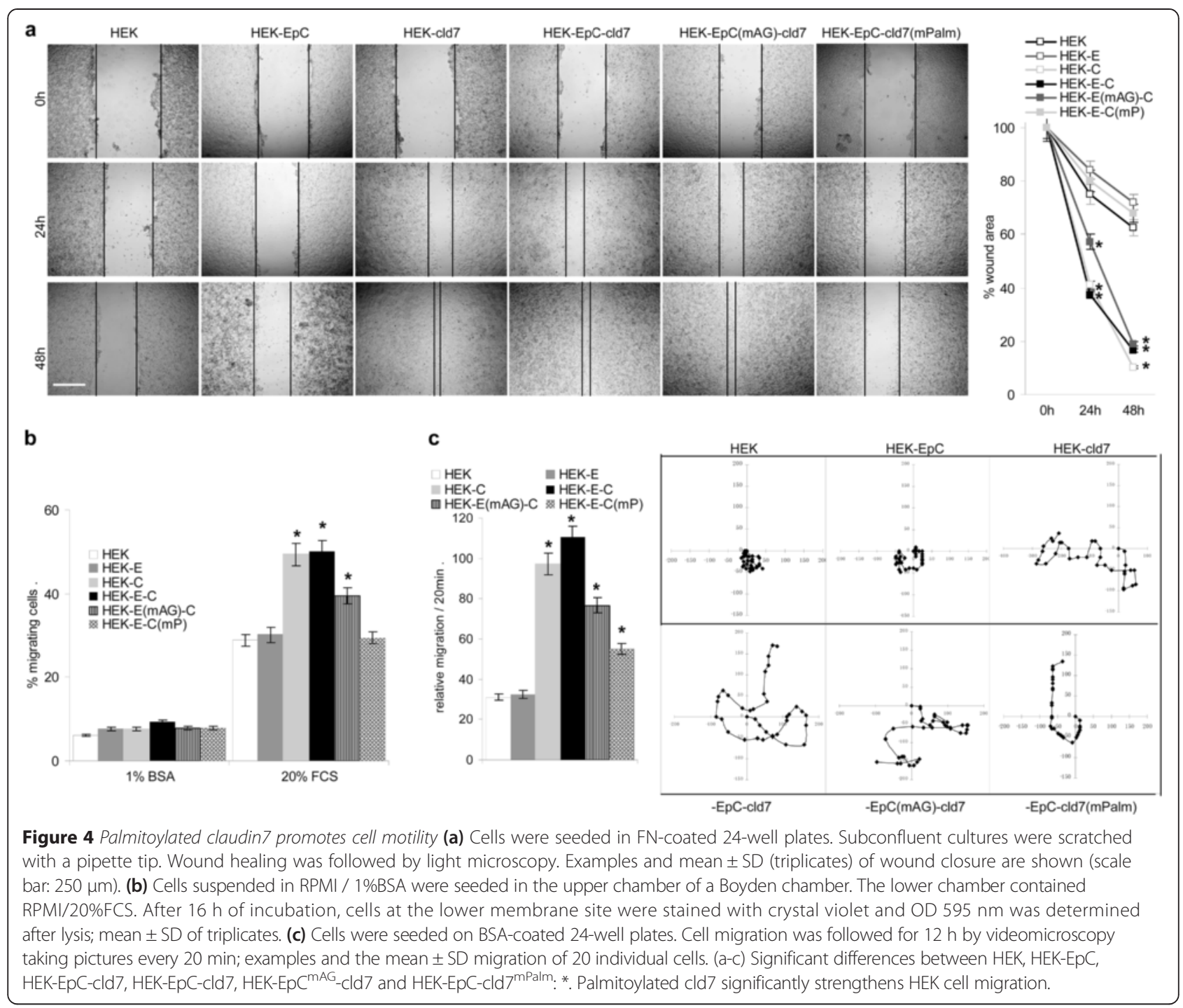

actin cytoskeleton (Figure 5a) is supported by ezrin, which associates with palmitoylated cld7. In fact, ezrin poorly associates with $\operatorname{cld} 7^{\mathrm{mPalm}}$ and the association is nearly abolished in the presence of a palmitoylation inhibitor (Figure 6b). As actin reorganization is not completely abolished in HEK-EpC-cld7 ${ }^{\text {mPalm }}$ cells, we speculated that the cld7-ezrin association is not a direct protein interaction, but is promoted by cld7-associated partner molecules. Besides with $\mathrm{EpC}$, cld7 co-immunoprecipitates with CD104, CD49e and CD166. Co-immunoprecipitation of cld7 with CD49e and CD166 is very weak in HEK$\mathrm{EpC}^{\mathrm{mAG}}$-cld7 and HEK-EpC-cld7 ${ }^{\mathrm{mPalm}}$ lysates. In antiezrin precipitates, recovery of $\mathrm{EpC}$ and cld7 is most strongly affected in HEK-EpC-cld $7^{\mathrm{mPalm}}$ and in antiCD49e and CD166 precipitates ezrin recovery is strongly reduced in HEK-EpC-cld $7^{\mathrm{mPalm}}$ (Figure 6c). These findings indicate that palmitoylated cld7 supports integrin activation. Activated integrins, in turn, associate via phosphorylated ezrin with the actin cytoskeleton. Supporting the mediator role of palmitoylated cld7, src did not co-immunoprecipitate with cld7 in HEK-EpC-cld $7^{\text {mPalm }}$ lysates. Co-immunoprecipitation of FAK and paxillin was not abolished, though reduced (Figure 6d). Thus, GEMlocated palmitoylated cld7 supports motility via the association with ezrin and integrins, where integrin activation contributes to increased motility.

Videomicroscopy pointed towards an additional contribution of $\mathrm{EpC}$ to cld7-promoted motility (Figure 4c). Cld7 contributes via the association with TACE to EpC cleavage (Figure 3f). As presenilin2 also resides in GEM [58], we speculated that the association of EpC with cld7 might facilitate the release of EpIC. Staining with anti-cld7 and anti-EpIC revealed, expectedly, stronger colocalization in the membrane of HEK-EpC-cld7 than HEK-EpC$\operatorname{cld} 7^{\mathrm{mPalm}}$ cells, but also more pronounced recovery of free EpIC in the cytoplasm. WB confirmed higher EpIC 


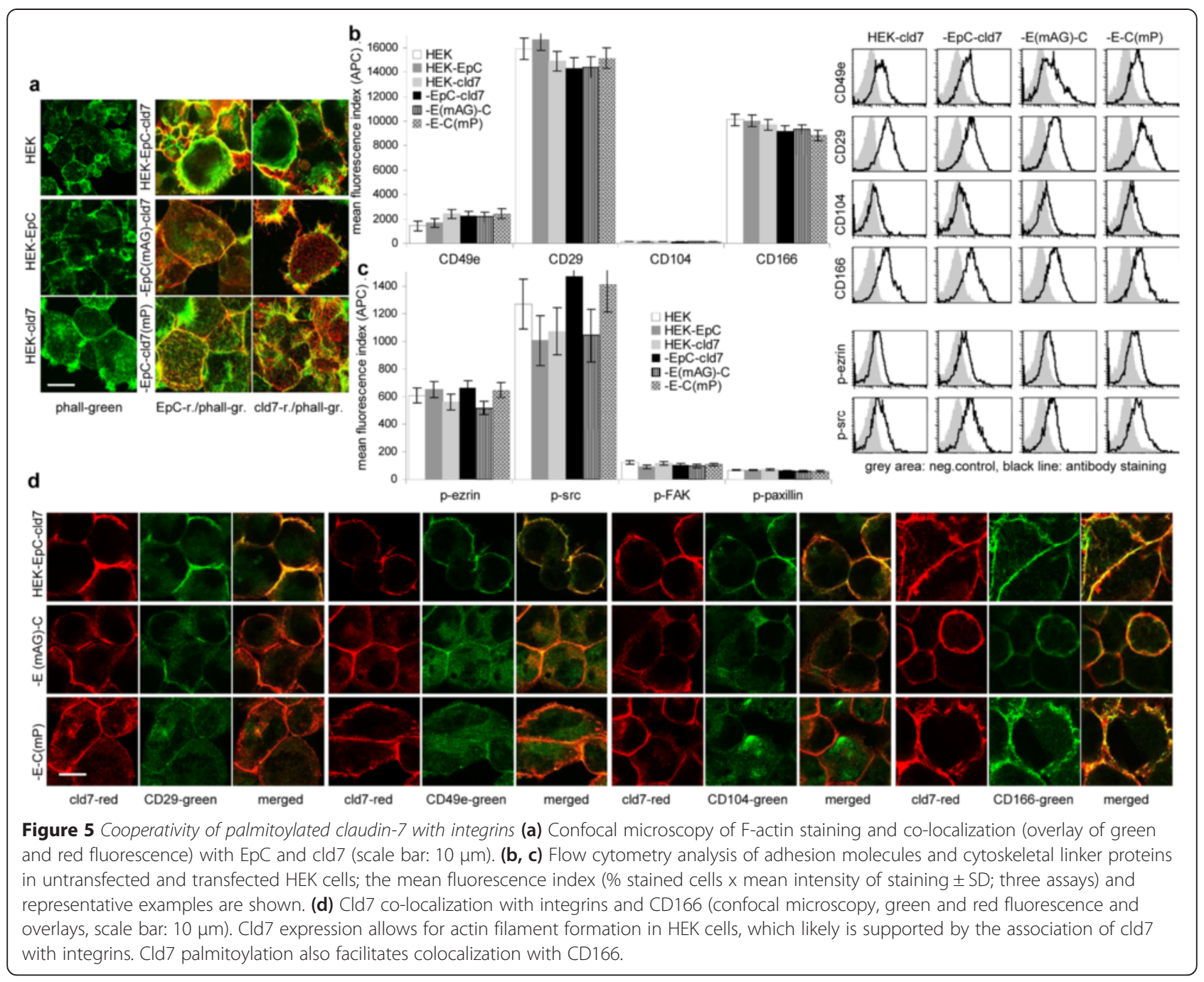

recovery in HEK-EpC-cld7 than in HEK-EpC lysates. On the

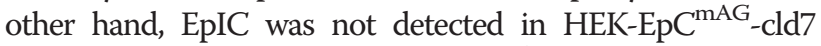
and very weakly in HEK-EpC-cld7 ${ }^{\mathrm{mPalm}}$ lysates (Figure 7a). This implies that palmitoylated cld7-associated, GEMlocated EpC becomes particularly susceptible for presenilin2. Nonetheless, presenilin2 co-immunoprecipitated also with $\operatorname{cld} 7^{\mathrm{mPalm}}$, but exclusively in heavy fractions (Figure $7 \mathrm{~b})$. Flow cytometry and WB confirmed unaltered $\beta$-catenin expression in HEK-cld7, -EpC-cld7, -EpC ${ }^{\text {mAG }}$ cld7 and -EpC-cld $7^{\mathrm{mPalm}}$ cells and only a minor reduction of presenilin2 in HEK-EpC-cld $7^{\mathrm{mPalm}}$ cells (Figure 7c, Additional file 4). Increased EpIC recovery in HEK-EpCcld7 cells was accompanied by slightly upregulated expression of vimentin, fibroblast growth factor (FGF), TGF $\beta$ and $\mathrm{N}$-cadherin. Upregulated $\mathrm{N}$-cadherin expression did not correlate with the generation of EpIC (HEK-EpC-cld7) and downregulation of E-cadherin in HEK-EpC-cld7 cells did not reach the level of statistical significance. Colocalization of FGF, N-cadherin and vimentin is less pronounced in HEK-EpC-cld $7^{\text {mPalm }}$ than HEK-EpC-cld7 cells
(Figure 7d, 7e), where palmitoylated cld7 supports EpIC generation. The pronounced EpIC generation by palmitoylated cld7 might strengthen expression of mesenchymal markers associated with a migratory phenotype. However, our findings point towards an engagement of additional components.

Taken together, cld7 promotes motility, which is partly due to the association of palmitoylated cld7 with p-ezrin and is supported by the association with integrins and CD166. Palmitoylated cld7 promotes the generation of EpIC, which contributes to motility by forcing mesenchymal protein expression.

\section{Palmitoylated claudin-7 supports invasiveness}

Cld7 repressing MMP3 [43], we wondered whether HEK-cld7 cells gain in motility, but concomitantly loose invasiveness.

HEK-cld7, HEK-EpC-cld7 and HEK-EpC ${ }^{\mathrm{mAG}}$-cld7 cells show pronounced matrigel invasion. Invasion of HEKEpC-cld $7^{\mathrm{mPalm}}$ resembles that of HEK, i.e. is strongly 


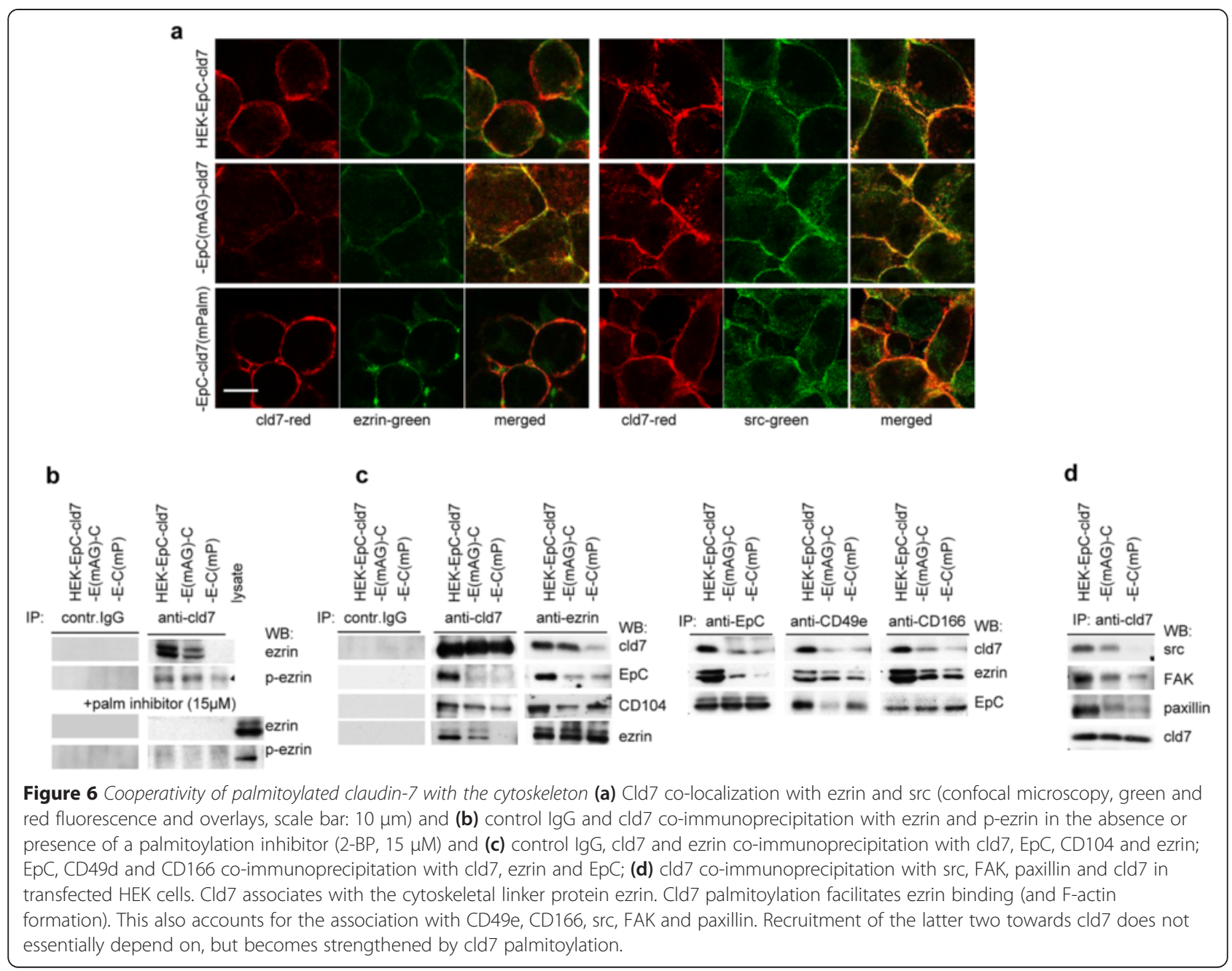

reduced (Figure 8a). This expectation-opposing finding prompted a search for MMP activity of untransfected and transfected HEK cells. HEK cells express MMP2, MMP3, MMP7, MMP9 and MMP13 at low, MMP14 at intermediate and CD147 at high level. MMP3, MMP7 and MMP9 expression is slightly reduced in cld7 expressing cells, but the reduction does not essentially correlate with cld7 palmitoylation (Figure 8b). Notably, cld7 does not colocalize with MMP3 and only palmitoylation-competent cld7 weakly colocalizes with MMP14 (Figure 8c).

As changes in protease expression could not explain increased invasiveness of HEK-EpC-cld7 cells, we speculated on pronounced protease activation, possibly due to the weak association of cld7 with MMP14. Zymography revealed low MMP2 and MMP9 activity in HEK and HEK-EpC cells, strongly increased activity in HEK-EpCcld7, but reduced activity in HEK-EpC-cld $7^{\text {mPalm }}$ cells (Figure 9a). Though this finding fits reduced invasiveness of HEK-EpC-cld $7^{\mathrm{mPalm}}$, we wondered, whether palmitoylated cld7 recruits CD147 (EMMPRIN) into GEM, which could contribute to MMP stabilization. Indeed,
anti-CD147 efficiently inhibits matrigel invasion of HEK-cld7, HEK-EpC-cld7 and HEK-EpC ${ }^{\text {mAG }}$-cld7 cells (Figure 9b). Furthermore, CD147 colocalizes and coimmunoprecipitates with cld7 in HEK-EpC-cld7 and HEK-EpC ${ }^{\mathrm{mAG}}$-cld7, but hardly in HEK-EpC-cld7 ${ }^{\mathrm{mPalm}}$ cells (Figure 9c, 9d, Additional file 3).

Though slightly affecting MMP3, MMP7 and MMP9 expression, cld7 fosters invasiveness via GEM-located MMP14, which holds MMP2 and MMP9 in membrane proximity. High CD147 expression likely promotes MMP induction.

In brief, palmitoylated cld7 poorly associates with $\mathrm{TJ}$ proteins. Instead, it is recruited into GEM, where it associates with the cytoskeleton, integrins, and integrin signaling partners, EpC and proteases. It promotes integrin and protease activation and $\mathrm{EpC}$ cleavage, supporting motility and invasion.

\section{Discussion}

Functional activities of claudins outside TJ are not well explored. This includes cld7, highly expressed in the 


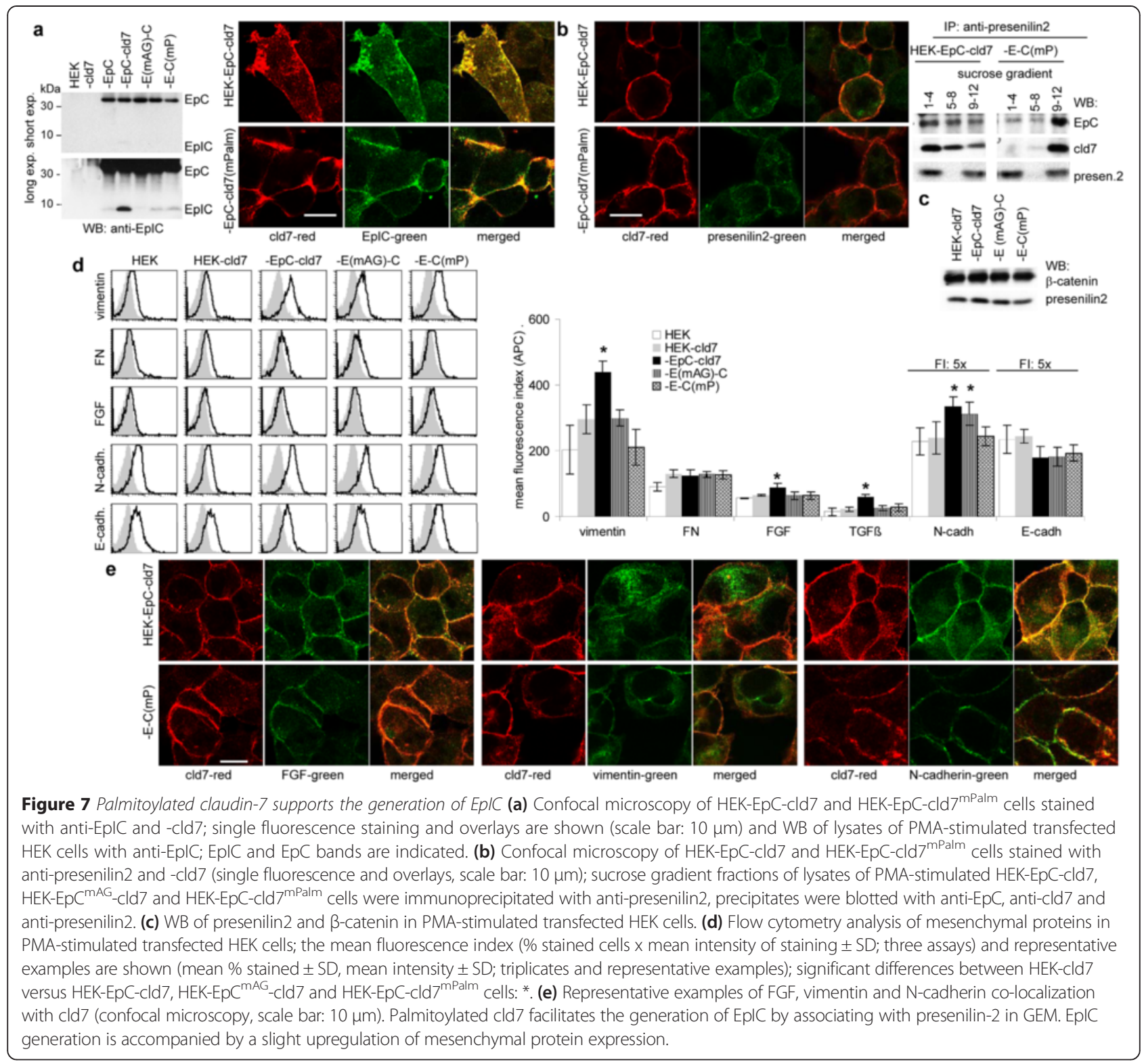

upper gastrointestinal tract and the liver, where it is not exclusively found in TJ $[6,8,9,28,29,59]$. Instead, cld7 associates in hepatocyte progenitors and gastrointestinal tumors with EpC that is not a TJ component $[36,40,59,60]$. Taking the importance of cld7 in organogenesis, cld $7^{\text {ko }}$ mice dying few days after birth [43], it becomes demanding to unravel TJ-independent cld7 activities. We approached the question of cld7 localization using easily transfectable HEK cells to generate a tool of cells stably expressing wt or mutated cld7 and/or EpC. Whereas single serine to alanine mutations was ineffective, a palmitoylation site mutation was accompanied by a striking shift in the membrane subdomain localization. Thus, we focused on palmitoylation defective cld7 and on mutated EpC that cannot bind cld7 [61]. The latter allowed differentiating between genuine cld7 activities and those requiring the association with $\mathrm{EpC}$.

\section{Cld7 palmitoylation and tight junctions}

In advance of discussing the impact of cld7 palmitoylation on membrane subdomain localization, it should be remembered that mutating AA184 and AA186 efficiently prevented cld7 palmitoylation and that palmitoylation at the C-terminal tail appears to be dominating, because only a very faint palmitoylation signal was detected in HEK-EpC-cld $7^{\text {mPalm }}$. The high efficacy of $\mathrm{cld} 7^{\mathrm{mPalm}}$ relocalization and striking differences in associating molecules are in line with the dominance of the C-terminal tail cld7 palmitoylation. 


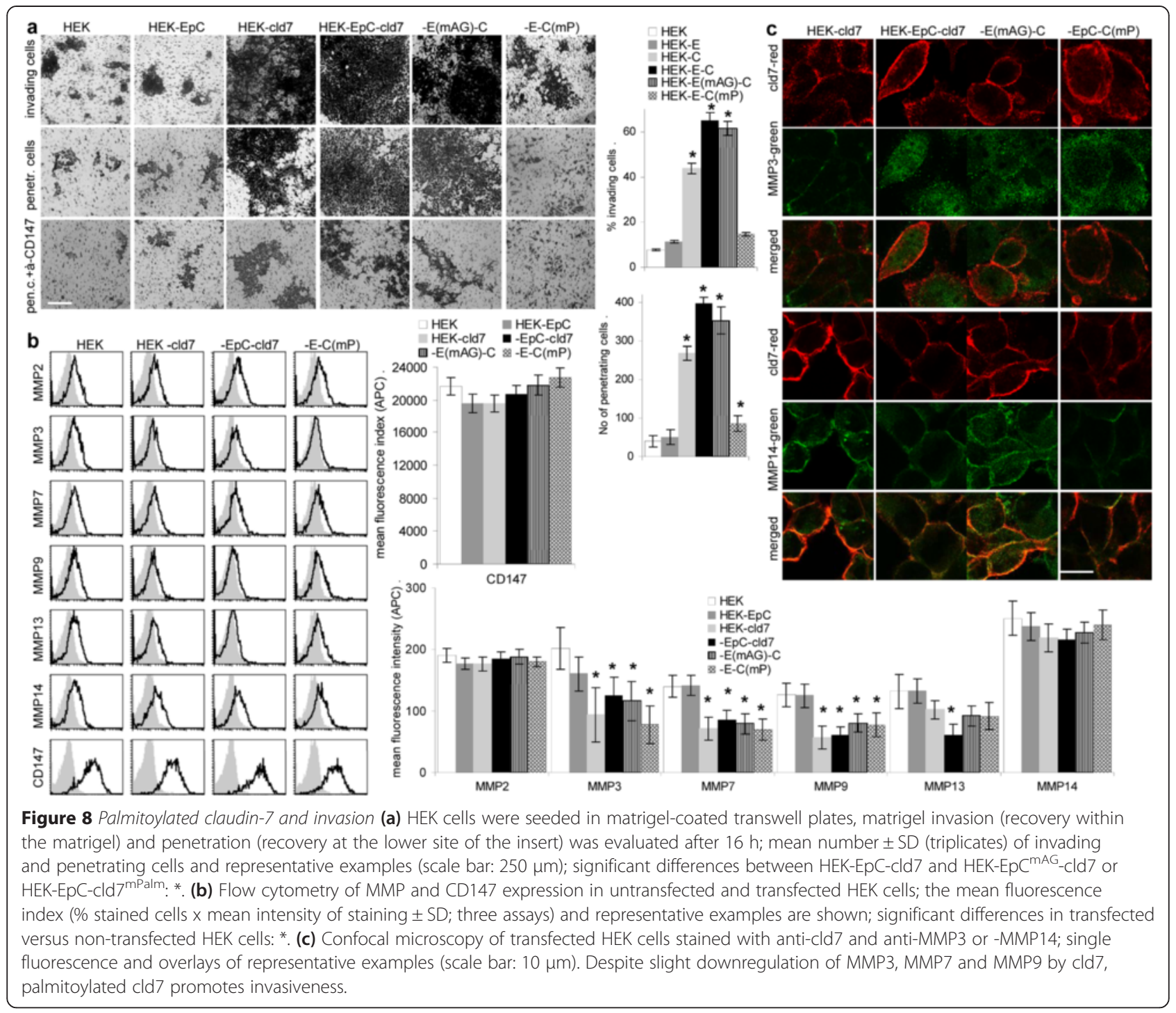

Palmitoylated cld7 is enriched in GEM, but is not recovered in TJ protein complexes. Cld7 poorly colocalizes and co-immunoprecipitates with cld3, cld4 and ZO-1, which strikingly differs from colocalization and co-immunoprecipitation of ZO-1 with cld3 and cld4. Instead, palmitoylation-deficient cld7 associates with TJ proteins. We have not explored, whether overexpression of cld $7^{\mathrm{mPalm}}$ in HEK cells suffices establishing classical TJ, as was described for a cld7 overexpressing epithelial like cell line [62]. However, HEK-EpCcld $7^{\mathrm{mPalm}}$ cells do not form tightly packed monolayers like epithelial cells and trogocytosis of membrane fractions from biotinylated HEK-EpC-cld $7^{\mathrm{mPalm}}$ by non-biotinylated HEK-EpC-cld7 ${ }^{\mathrm{mPalm}}$ was not in support of "kissing points" (data not shown). On the other hand, only palmitoylated cld7 associates with EpC.

Palmitoylation supports anchoring in GEM [16,17]. GEM microdomains are signaling platforms facilitating attachment of cytoplasmic kinases [16,18,19]. GEM also are prone for internalization [21-24]. This is a sequel of the attachment of complexes that promote scission, fission and early endosome traffic towards multivesicular bodies, where proteins become ubiquitinated and degraded in the proteasome [63]. Alternatively, the multivesicular bodies are transported back to the plasma membrane and the intraluminal vesicles are released as exosomes [64]. Notably, claudins were recovered in internal vesicles colocalizing with syntaxin4 [65], but did not reintegrate into TJ. Syntaxin4 being one of the components for vesicular transport [66], we suggest an independent route of trafficking of TJ- versus GEM-integrated claudins. In fact, colon carcinoma lines secrete two populations of exosomes, only exosomes from the basolateral region contain the EpC-cld7 complex [59]. Furthermore, the association with $\mathrm{EpC}$ hampers the integration of cld7 and via cld7 the integration of cld1 into TJ [60]. 


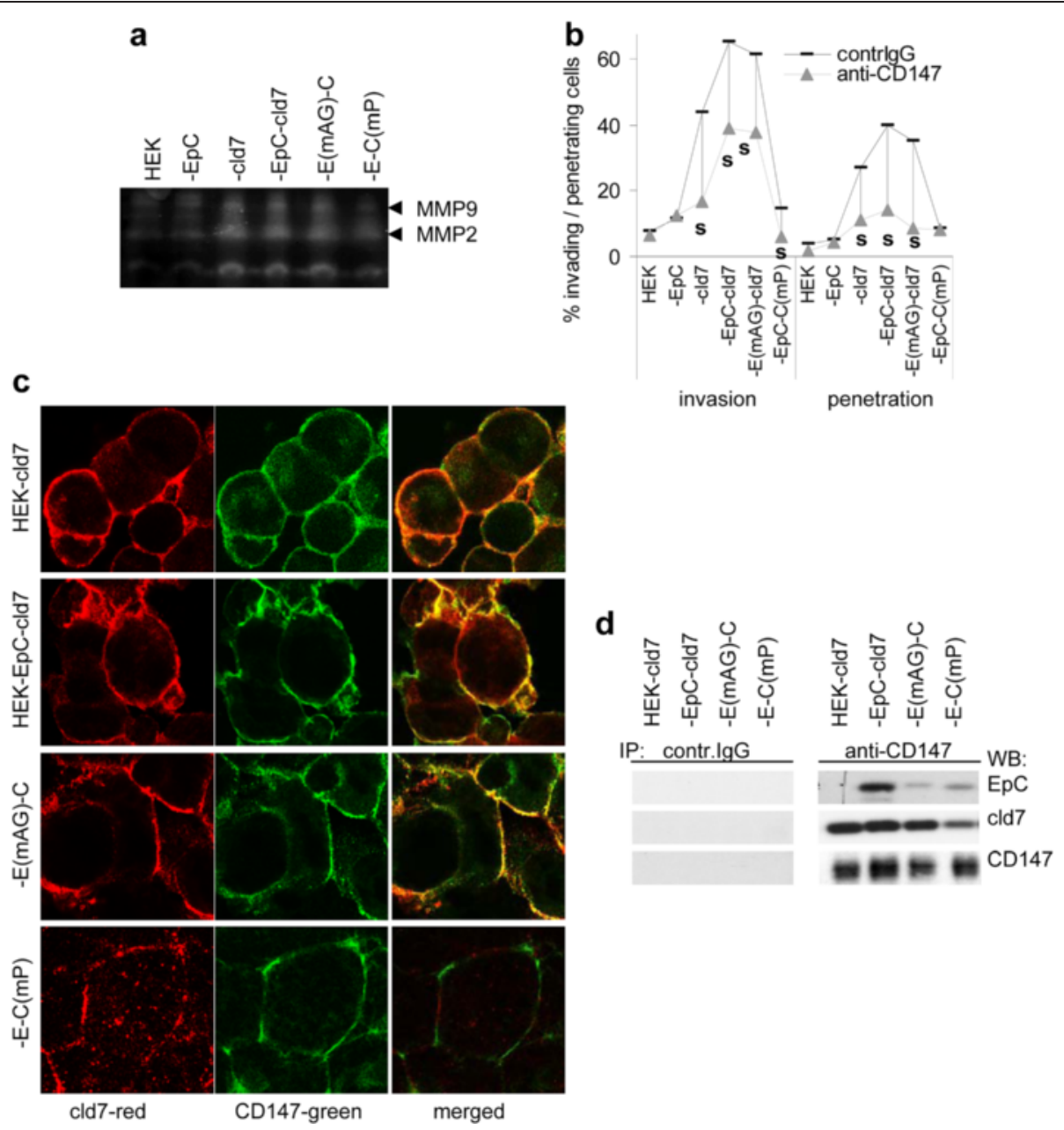

Figure 9 Palmitoylated claudin-7, MMP activity and CD147 (a) Zymography of HEK, HEK-EpC, HEK-cld7, HEK-EpC-cld7 and HEK-EpC ${ }^{\mathrm{mAG}}$-cld7 or HEK-EpC-cld7 ${ }^{\mathrm{mPalm}}$ supernatants. (b) HEK cells were seeded in matrigel-coated transwell plates in the presence of anti-CD147 (10 $\left.\mu \mathrm{g} / \mathrm{ml}\right)$ (a representative example is included in Figure 8a); invasion and penetration was evaluated as above; significant inhibition by anti-CD147: s. (c)

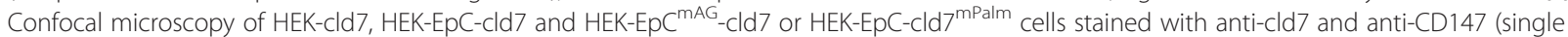
fluorescence and overlays, scale bar: $10 \mu \mathrm{m}$ ). (d) HEK-cld7, HEK-EpC-cld7, HEK-EpC ${ }^{\mathrm{mAG}}$-cld7 and HEK-EpC-cld7 ${ }^{\mathrm{mPalm}}$ lysates were precipitated with control IgG or anti-CD147; after SDS-PAGE and transfer precipitates were blotted with anti-EpC, anti-cld7 and anti-CD147. Palmitoylated cld7-promoted invasiveness likely is supported by associated MMP14 and CD147, which can strengthen MMP2 and MMP9 activation and expression.

Palmitoylation-deficiency of cld7 had significant bearing on associating molecules and their activation state. A strikingly reduced number of proteins coimmunoprecipitated with cld7 in lysates of HEK-EpC$\mathrm{cld} 7^{\mathrm{mPalm}}$ and phosphorylation of co-immunoprecipitating proteins was additionally impaired. The number of molecules co-immunoprecipitating with anti-cld7 from HEK$\mathrm{EpC}^{\mathrm{mAG}}$-cld7 lysates was also reduced, though the reduction was less pronounced. These findings indirectly confirm the direct association of $\mathrm{EpC}$ with cld7 [35] and support a dominating role of palmitoylation-competent cld7 in associated molecule activation. It is particularly worthwhile noting that only palmitoylation-competent cld7 abundantly associates with vesicular transport molecules. It remains to be explored, whether this translates into a central role of cld7 in exosome composition and release.

Taken together, the membrane subdomain localization of cld7 is dictated by palmitoylation. This has most severe consequences on associating molecules and their activation state. Building on this, we asked for functional consequences.

\section{Palmitoylated claudin-7 prohibits adhesion and supports motility}

The striking impact of cld7 palmitoylation on the redistribution into GEM and on the association with (phosphorylated) GEM-located molecules suggests that palmitoylated cld7 fulfills TJ-independent activities. We had described that tetramer formation and homophilic EpC binding [67] 
is distorted by the association with cld7 [56]. We now reported that the cld7-mediated inhibition of homophilic $\mathrm{EpC}$ binding depends on cld7 palmitoylation and the GEM localization. The elegant study by Wu et al. [60] also reported that $\mathrm{EpC}$ interferes with the integration of cld1 and cld7 in TJ and supports recruitment into vesicles. These findings are in line with our observations, which suggest that cld7 palmitoylation is the prime cause, followed by the association with EpC. GEM-located TACE [68], which cuts transmembrane molecules in membrane proximal regions [69], supports the interference of cld7 with homophilic EpC binding by the liberation of EpEx. Thus, palmitoylated cld7 inhibits EpC-mediated cell-cell adhesion by prohibiting tetramer formation and by promoting EpEx liberation.

Palmitoylated cld7 also actively supports cell motility that is accompanied by organization of the disordered actin cytoskeleton in HEK cells [57]. GEM-located, palmitoylated cld7 associates with CD166 and $\alpha 5$, the latter being the most abundantly expressed integrin in HEK cells [57]. The cld7 colocalization with $\alpha 5$ and CD166 in GEM suffices for src, FAK and paxillin association and phosphorylation. Importantly, palmitoylated cld7 associates with phosphorylated ezrin, which can explain the striking consequences on HEK cell motility and actin cytoskeleton organization. As outlined above, cld7 also cooperates with TACE, which also cleaves the cellmatrix adhesion molecule CD166 [39,70,71]. It remains to be explored, whether cleavage of CD166 indirectly supports motility by breaking adhesive cell contacts.

Palmitoylated cld7 additionally contributes to motility by facilitating EpIC generation. By a change in conformation after EpEx cleavage, presenilin2 can bind and liberate the cotranscription factor EpIC [39]. Though EpIC generation may not essentially depend on cld7 coexpression [39], EpC recruitment into GEM via palmitoylated cld7 strengthens the efficacy of EpIC generation, which is accompanied by increased expression of the mesenchymal proteins FGF, TGF $\beta$ and N-cadherin. The shift towards mesenchymal protein expression could strengthen motility.

Though the association of palmitoylated cld7 with $\mathrm{EpC}$ does not suffice for a full-fledged shift towards EMT, our findings are quite distinct from a recent publication on cld7-induced MET, which is accompanied by rab25 upregulation [72]. Rab25 can be a tumor suppressor in colon cancer [73], but promotes tumor growth in several different cancer types [74]. Interestingly, in hormone receptor negative breast cancer rab25 expression is very low, but the small subpopulation of cancer initiating cells express rab25 [75]. Based on this finding, we suggest that palmitoylated cld7 and the vesicle transporter rab25 support cancer-initiating cell activities, possibly via the transfer of exosomes. Our suggestion is in line with the central importance of rab molecules in vesicle traffic and exosome release [76], the delivery of cld7-EpC complex expressing exosomes by colorectal cancer cells and the abundant association of vesicle traffic-associated molecules with palmitoylation-competent cld7.

Despite the need to experimentally support the latter hypothesis, our data demonstrate an active contribution of palmitoylated cld7 to cell motility, which is based on the GEM-location-dependent association with $\mathrm{EpC}$, integrins, CD166, cytoskeletal linker and signaling molecules.

\section{Claudin7 and proteases}

Cld7 can repress MMP3 [43], which could hamper invasiveness. Opposing our expectation, HEK-cld7 cells are strongly invasive. HEK cells express MMP3 and MMP7 at an intermediate level. Independent of cld7 palmitoylation, both proteases are slightly downregulated in HEKcld7 cells. Cld7 expression did not affect expression of other MMPs in HEK cells. On the other hand, gelatinolytic activity of HEK-cld7 cells was increased. Palmitoylated cld7 colocalizes with MMP14, which supports retention of MMP2 and MMP9 close to the plasma membrane. Furthermore, HEK cells highly express CD147 that stimulates MMP expression in neighboring cells $[77,78]$. CD147 colocalizes and co-immunoprecipitates with palmitoylated cld7 and thereby can contribute to cld7promoted invasiveness. This interpretation is supported by the finding that anti-CD147 strongly inhibits HEK-cld7 and HEK-EpC-cld7, but not weak HEK-EpC-cld7 $7^{\text {mPalm }}$ cell invasion.

Taken together, in HEK cells a repression of MMP3 by cld7 has no bearing on invasiveness, as palmitoylated cld7 supports invasion by associating with MMP14 and CD147.

\section{Conclusions}

The (patho)physiological relevance of the EpC-cld7 complex was demonstrated repeatedly, most convincing by the death of $\mathrm{EpC}^{\mathrm{ko}}$ mice [41,42], by the shift of cld1 and cld7 towards TJ in $\mathrm{EpC}^{\mathrm{kd}}$ cells [60], by the pronounced recovery of GEM-located cld7-EpC-CD44v6-Tspan8 complexes in colorectal cancer $[36,52]$ and by the metastasissupporting activity of the EpC-cld7 complex in pancreatic and colorectal cancer [35,36,38,52,61]. We here demonstrated that under physiological conditions, i.e. without chemical or antibody crosslinking, a considerable part of cld7 is palmitoylated and that palmitoylation drives cld7 into GEM, but prohibits integration into TJ. In GEM, palmitoylated cld7 associates with adhesion molecules, proteases, cytoskeletal linker and signal transduction molecules, which supports motility and invasion. Though the reason for the preferential palmitoylation state of cld7 remains to be explored, palmitoylated cld7 exhibits TJ opposing activities, which should be taken into account considering the role of cld7 in development and metastasis. 


\section{Material and methods} Cell lines and transfectants

HEK293 cells [79] cells were transfected with rat EpC cDNA and cotransfected with rat cld7 cDNA using the pcDNA3.1 vector that carries either the neomycin or the hygromycin resistance cDNA. Where indicated, the cld7 cDNA was mutated at the serine residues AA33, AA69, AA87, AA172, AA204, AA206 and AA207, exchanging serine by alanine (HEK-EpC-cld $7^{\mathrm{mSxx}}$ ) and with cld7 cDNA, where the palmitoylation site was destroyed by mutations at AA184 and AA186 (HEK-EpC-cld $7^{\text {mPalm}}$ ). The EpC cDNA was mutated at AA279 and AA282, which are located in the transmembrane region and account for the EpC-cld7 association (HEK-EpC ${ }^{\mathrm{mAG}}$-cld7) (Additional file 1) and cDNA inserts were sequenced. Cells were transfected with lipofectamin and cultured in RPMI $/ 10 \%$ FCS plus $500 \mu \mathrm{g} / \mathrm{ml}$ G418 and/or $150 \mu \mathrm{g} / \mathrm{ml}$ hygromycin. Transfection efficacy, evaluated by WB and flow cytometry, ranged between $>90 \%$ to $98 \%$.

\section{Antibodies and chemicals}

Additional file 5.

\section{Sucrose density gradient centrifugation}

Cell lysates in $2.5 \mathrm{M}$ sucrose were overlaid by a continuous sucrose gradient $(0.25 \mathrm{M}-2 \mathrm{M})$ and centrifuged $(15 \mathrm{~h}, 150000 \mathrm{~g})$ and twelve $1 \mathrm{ml}$ fractions were collected. Proteins located in GEM are recovered in fractions $2-4$.

\section{In vitro kinase assay}

Immune complexes were suspended in lysis buffer containing a protease inhibitor mix. After centrifugation, beads were resuspended in $30 \mu \mathrm{l}$ kinase assay buffer, $10 \mu \mathrm{Ci}\left[{ }^{32} \mathrm{P}\right] \gamma$-ATP and incubated $\left(15 \mathrm{~min}, 37^{\circ} \mathrm{C}\right)$, stopping the reaction by $10 \mu \mathrm{l}$ non-reducing $6 \mathrm{x}$ Laemmli buffer. SDS-PAGE was followed by autoradiography.

\section{IP, Western blot (WB)}

Lysates (30 min, $4^{\circ} \mathrm{C}$, HEPES buffer, $1 \%$ Lubrol or $1 \%$ TritonX-100, $1 \mathrm{mM}$ PMSF, $1 \mathrm{mM} \mathrm{NaVO}$, $10 \mathrm{mM} \mathrm{NaF}$, protease inhibitor mix) were centrifuged (13000 g, $\left.10 \mathrm{~min}, 4^{\circ} \mathrm{C}\right)$, mixed with antibody $\left(1 \mathrm{~h}, 4^{\circ} \mathrm{C}\right)$ and incubated with ProteinG-Sepharose $(1 \mathrm{~h})$. For the analysis of released EpEx (EpC extracellular domain), culture supernatant were depleted of exosomes [80]; supernatant were 10-times concentrated. Washed complexes/lysates, dissolved in Laemmli buffer, were resolved in 10\%-12\% SDS-PAGE. For the recovery of EpIC, Tris-Tricine and 16\% SDS-PAGE was used under non-reducing condition. After protein transfer, blocking, blotting with antibodies, blots were developed with ECL.

\section{Palmitoylation assay}

Palmitoylation of cld7 was determined using the IP-ABE method [81]. In brief, cultured cells were lysed in the presence of N-ethylmaliemide (NEM), using lysis buffer (LB) $(50 \mathrm{mM}$ NEM, 1\% Triton-X-100, 1x protease inhibitor cocktail, $1 \mathrm{mM}$ phenylmethanesulfonylfluoride (PMSF)) for irreversibly blocking unmodified thiol groups. After an IP with anti-cld7, G-Sepharose coupled precipitates where incubated with hydroxylamine (HAM) buffer (1 h, room temperature, LB pH7.2, 1 M HAM) for specific cleavage and unmasking of the palmitoylated cysteine thiol group. Thereafter samples were incubated in biotinBMCC buffer (LB pH 6.2, $1 \mu \mathrm{M}$ biotin-BMCC, $1 \mathrm{~h}, 4^{\circ} \mathrm{C}$ ) for selective labeling of the palmitoylated cysteines. Samples were eluted in LB pH7.5 followed by SDS-PAGE and WB with streptavidin-HRP.

\section{MALDI-TOF analysis}

After SDS-PAGE, gels were stained. Protein digestion, sample preparation, MALDI-TOF fingerprint analysis, post-source decay fragmentation analysis and database searches were performed as described [82].

\section{Flow-cytometry}

Flow-cytometry followed routine procedures. In brief, $1-2.5 \times 10^{5}$ cells were seeded in 96 -well plates. After washing with $\mathrm{PBS} / 1 \% \mathrm{BSA}$, cells were incubated with the primary antibody $\left(2-10 \mu \mathrm{g} / \mathrm{ml}, 40 \mu \mathrm{l}, 30 \mathrm{~min}, 4^{\circ} \mathrm{C}\right)$. Cells were washed 3-times with $\mathrm{PBS} / 1 \% \mathrm{BSA}$ and incubated with a secondary dye-labeled antibody at predetermined concentration. Negative controls were only incubated with the secondary dye-labeled antibody $\left(40 \mu \mathrm{l}, 30 \mathrm{~min}, 4^{\circ} \mathrm{C}\right)$. For intracellular staining, cells were fixed (PBS/1\% formaldehyde, $30 \mathrm{~min}, 4^{\circ} \mathrm{C}$ ) and permeabilized (PBS/0.5\% Tween-20, $20 \mathrm{~min}, 4^{\circ} \mathrm{C}$ ). Samples were processed in a FACS-Calibur and evaluated with the CellQuest program. Data are presented as a weighted score, the fluorescence index (FI) representing the percentage stained cells $\times$ the mean fluorescence intensity above background.

\section{ELISA}

For the recovery of EpEx, cells were cultured in serumfree medium for $48 \mathrm{~h}$. Supernatants were centrifuged, concentrated and adjusted to $100 \mu \mathrm{g} / \mathrm{ml}$, evaluating the amount of EpEx by a direct ELISA coating the wells with culture supernatant and proceeding with a standard protocol using anti-rat EpC (D5.7) for detection.

\section{Confocal microscopy}

Cells on glass-slides were fixed (4\% paraformaldehyde, $20 \mathrm{~min}$ on ice), permeabilized (1\% Triton-X100, $4 \mathrm{~min}$, on ice), blocked (PBS/1\% gelatin, $30 \mathrm{~min}$, on ice), incubated with primary antibody (60 min, on ice), washed, incubated with fluorochrome-conjugated secondary antibody (60 min, 
on ice), blocked (IgG with irrelevant specificity of the same species as the primary antibody), incubated with a second, dye-labeled primary antibody and washed. Slides were mounted in Elvanol. Digitized images were generated using a Leica LMS780 microscope and the Carl Zeiss Vision software for evaluation. The Z-stack offers 30 positions through the depth of the cell. All pictures were taken at Z-stack 14-16. Depending on the quality of the antibody and the density of marker expression, the intensity for the green channel varied between 700-900 master gain values and for the red channel between 500-750 master gain values. The photosystem automatically generates the single fluorescence and overlay pictures.

\section{Cell adhesion}

CFSE-labeled cells were seeded on a cell monolayer in 96-well plates. After washing, adherent cells were lysed, evaluating fluorescence intensity photometrically. Adhesion is presented as percent seeded cells.

\section{Migration}

Cells, in the upper part of a Boyden chamber (RPMI / $0.1 \% \mathrm{BSA}$ ), were separated from the lower part (RPMI / $20 \%$ FCS) by $8 \mu \mathrm{m}$ pore size polycarbonate-membranes. After $16 \mathrm{~h}$, the lower membrane side was stained (crystal-violet), measuring OD595 after lysis. Migration is presented as percentage input cells. In an in vitro wound healing assay, a subconfluent monolayer was scratched with a pipette tip. Wound closure was controlled by light microscopy. For videomicroscopy, cells $\left(5 \times 10^{4}\right)$ were seeded on matrix protein-coated 24-well plates. Plates were placed under an Olympus IX81 inverse microscope with an $\mathrm{Hg} / \mathrm{Xe}$ lamp, an incubation chamber $\left(37^{\circ} \mathrm{C}, 5 \%\right.$ $\mathrm{CO}_{2}$ ), a CCD camera (Hamamatsu) and a ScanR acquisition soft ware (Olympus, Hamburg, Germany). Two pictures (20-fold magnification)/chamber ( $2 \mathrm{~ms}$ exposure) were taken every $20 \mathrm{~min}$ for $12 \mathrm{~h}$. Migration was quantified according to Manual_tracking plugin running in the open-source software Image J.

\section{Statistics}

All experiments were repeated at least 3 times. Mean values of triplicates or for videomicroscopy of 20 individual cells or of, at least 3 independent experiments are presented. Significance was evaluated by the two tailed Student's $t$-test. P-values $<0.05$ were considered significant.

\section{Additional files}

Additional file 1: Primers.

Additional file 2: The impact of cld7 serines on membrane subdomain localization and the association with EpCAM.
Additional file 3: MALDI-TOF analysis of molecules co-immunoprecipitating with cld7.

Additional file 4: The impact of cld7 palmitoylation on cld7 phosphorylation, presenilin2 and $\beta$-catenin expression.

Additional file 5: Antibodies and chemicals.

\section{Abbreviations}

cld7: claudin-7; cld $7^{\mathrm{mPalm}}$ : cld7 with a mutated palmitoylation site; $\mathrm{cld} 7^{\mathrm{mSxx}}$ : cld7 with serine exchange by alanine at the indicated AA; EpC: EpCAM; EpC ${ }^{A G}$ : EpC with a point mutation at AA279 and AA282, which prohibits cld7 binding; EpEx: EpC extracellular domain; EplC: EpC intracellular domain; GEM: glycolipid-enriched membrane domains; HEK: HEK293 cells; HEK-EPC: HEK cells transfected with EpC; HEK-cld7: HEK cells transfected with cld7; HEK-EpC ${ }^{\text {mAG }}$ : HEK cells transfected with EpC ${ }^{\text {mAG }}$; HEK-cld7 $7^{\text {mPalm}}$ : HEK cells transfected with cld $7^{\mathrm{mPalm}}$; m: mutated; palm: palmitoylation; TJ: tight junctions.

\section{Competing interests}

The authors declare that they have no competing interests.

\section{Authors' contribution}

SH, WM performed and analyzed experiments, FT and MZ helped with experiments and analysis, FT and MZ wrote the manuscript. All authors read and approved the final manuscript.

\section{Acknowledgement}

This investigation was supported by the Deutsche Krebshilfe (MZ).

Received: 6 January 2015 Accepted: 21 April 2015

Published online: 09 June 2015

\section{References}

1. Tsukita S, Furuse M. Overcoming barriers in the study of tight junction functions: from occludin to claudin. Genes Cells. 1998;3:569-73.

2. Schneeberger EE, Lynch RD. The tight junction: a multifunctional complex. Am J Physiol Cell Physiol. 2004;286:C1213-28.

3. Singh AB, Sharma A, Dhawan P. Claudin family of proteins and cancer: an overview. J Oncol. 2010;2010:541957.

4. Schulzke JD, Günzel D, John L, Fromm M. Perspectives on tight junction research. Ann NY Acad Sci. 2012;1257:1-19.

5. Kwon MJ. Emerging roles of claudins in human cancer. Int J Mol Sci. 2013;14:18148-80.

6. Ding L, Lu Z, Lu Q, Chen YH. The claudin family of proteins in human malignancy: a clinical perspective. Cancer Manag Res. 2013;5:367-75.

7. Escudero-Esparza A, Jiang WG, Martin TA. The Claudin family and its role in cancer and metastasis. Front Biosci. 2011;16:1069-83.

8. Lal-Nag M, Morin PJ. The claudins. Genome Biol. 2009;10:235.

9. Rahner C, Mitic LL, Anderson JM. Heterogeneity in expression and subcellular localization of claudins 2, 3, 4, and 5 in the rat liver, pancreas, and gut. Gastroenterology. 2001;120:411-22.

10. Li WY, Huey CL, Yu AS. Expression of claudin-7 and -8 along the mouse nephron. Am J Physiol Renal Physiol. 2004;286:F1063-71.

11. Van Itallie CM, Gambling TM, Carson JL, Anderson JM. Palmitoylation of claudins is required for efficient tight-junction localization. J Cell Sci. 2005;118(Pt 7):1427-36

12. Butt $A M$, Khan IB, Hussain M, Idress M, Lu J, Tong Y. Role of post translational modifications and novel crosstalk between phosphorylation and O-beta-GlcNAc modifications in human claudin-1, -3 and -4 . Mol Biol Rep. 2012;39:1359-69.

13. Stipp CS, Kolesnikova TV, Hemler ME. Functional domains in tetraspanin proteins. Trends Biochem Sci. 2003;28:106-12.

14. Chini B, Parenti M. G-protein-coupled receptors, cholesterol and palmitoylation: facts about fats. J Mol Endocrinol. 2009:42:371-9.

15. Zhang W, Trible RP, Samelson LE. LAT palmitoylation: its essential role in membrane microdomain targeting and tyrosine phosphorylation during $T$ cell activation. Immunity. 1998:9:239-46.

16. Stepanek O, Draber P, Horejsi V. Palmitoylated transmembrane adaptor proteins in leukocyte signaling. Cell Signal. 2014;26:895-902. 
17. Diaz-Rohrer BB, Levental KR, Simons K, Levental I. Membrane raft association is a determinant of plasma membrane localization. Proc Natl Acad Sci U S A. 2014;111:8500-5.

18. Yáñez-Mó M, Barreiro O, Gordon-Alonso M, Sala-Valdés M, Sánchez-Madrid F. Tetraspanin-enriched microdomains: a functional unit in cell plasma membranes. Trends Cell Biol. 2009;19:434-46.

19. Ingley E. Src family kinases: regulation of their activities, levels and identification of new pathways. Biochim Biophys Acta 2008; 1784:56-65

20. Lajoie P. Nabi IR Lipid rafts, caveolae, and their endocytosis. Int Rev Cell Mol Biol. 2010;282:135-63.

21. Marchese A. Endocytic trafficking of chemokine receptors. Curr Opin Cell Biol. 2014;27:72-7.

22. Stahl PD, Barbieri MA. Multivesicular bodies and multivesicular endosomes: the "ins and outs" of endosomal traffic. Sci STKE. 2002;2002:32.

23. Rodgers W, Zavzavadjian J. Glycolipid-enriched membrane domains are assembled into membrane patches by associating with the actin cytoskeleton. Exp Cell Res. 2001;267:173-83.

24. Yeaman C, Grindstaff KK, Hansen MD, Nelson WJ. Cell polarity: Versatile scaffolds keep things in place. Curr Biol. 1999;9:515-7.

25. Ikari A, Ito M, Okude C, Sawada H, Harada H, Degawa M, et al. Claudin-16 is directly phosphorylated by protein kinase A independently of a vasodilator-stimulated phosphoprotein-mediated pathway. J Cell Physiol. 2008;214:221-9.

26. French AD, Fiori JL, Camilli TC, Leotlela PD, O'Connell MP, Frank BP, et al. PKC and PKA phosphorylation affect the subcellular localization of claudin-1 in melanoma cells. Int J Med Sci. 2009;6:93-101.

27. Sjö A, Magnusson KE, Peterson KH. Protein kinase $\mathrm{C}$ activation has distinct effects on the localization, phosphorylation and detergent solubility of the claudin protein family in tight and leaky epithelial cells. J Membr Biol. 2010;236:181-9.

28. Findley MK, Koval M. Regulation and roles for claudin-family tight junction proteins. IUBMB Life. 2009;61:431-7.

29. Van Itallie CM, Anderson JM. Claudin interactions in and out of the tight junction. Tissue Barriers. 2013;1:e25247.

30. Kovalenko OV, Yang XH, Hemler ME. A novel cysteine cross-linking method reveals a direct association between claudin-1 and tetraspanin CD9. Mol Cell Proteomics. 2007;6:1855-67.

31. Stamataki Z, Grove J, Balfe P, McKeating JA. Hepatitis C virus entry and neutralization. Clin Liver Dis. 2008;12:693-712.

32. Harris HJ, Davis C, Mullins JG, Hu K, Goodall M, Farquhar MJ, et al. Claudin association with CD81 defines hepatitis C virus entry. J Biol Chem. 2010;285:21092-102.

33. Tanaka M, Kamata R, Sakai R. Phosphorylation of ephrin-B1 via the interaction with claudin following cell-cell contact formation. EMBO J. 2005;24:3700-11.

34. Tanaka M, Kamata R, Sakai R. EphA2 phosphorylates the cytoplasmic tail of Claudin-4 and mediates paracellular permeability. J Biol Chem. 2005;280:42375-82.

35. Ladwein M, Schmidt DS, Schnölzer M, Kempf T, Pape UF, Langbein L, et al. The cell-cell adhesion molecule EpCAM associates with the tight junction protein claudin 7. Exp Cell Res. 2005;309:345-57.

36. Kuhn $S$, Koch $M$, Nübel $T$, Ladwein $M$, Antolovic $D$, Klingbeil $P$, et al. A complex of EpCAM, claudin-7, CD44 variant isoforms, and tetraspanins promotes colorectal cancer progression. Mol Cancer Res. 2007:5:553-67.

37. Okada T, Nakamura T, Watanabe T, Onoda N, Ashida A, Okuyama R, et al. Coexpression of EpCAM, CD44 variant isoforms and claudin-7 in anaplastic thyroid carcinoma. PLoS One. 2014;9:e94487.

38. Thuma F, Zöller M. EpCAM-associated claudin-7 supports lymphatic spread and drug resistance in rat pancreatic cancer. Int J Cancer. 2013;133:855-66.

39. Maetzel D, Denzel S, Mack B, Canis M, Went P, Benk M, et al. Nuclear signalling by tumour-associated antigen EpCAM. Nat Cell Biol. 2009;11:162-71.

40. Yovchev Ml, Grozdanov PN, Zhou H, Racherla H, Guha C, Dabeva MD. Identification of adult hepatic progenitor cells capable of repopulating injured rat liver. Hepatology. 2008:47:636-47.

41. Mueller JL, McGeough MD, Peña CA, Sivagnanam M. Functional consequences of EpCam mutation in mice and men. Am J Physiol Gastrointest Liver Physiol. 2014;306:G278-88.
42. Lei Z, Maeda T, Tamura A, Nakamura T, Yamazaki Y, Shiratori H, et al. EpCAM contributes to formation of functional tight junction in the intestinal epithelium by recruiting claudin proteins. Dev Biol. 2012;371:136-45.

43. Ding L, Lu Z, Foreman O, Tatum R, Lu Q, Renegar R, et al. Inflammation and disruption of the mucosal architecture in claudin-7-deficient mice. Gastroenterology. 2012;142:305-15.

44. Le Naour F, Zöller M. The tumor antigen EpCAM: tetraspanins and the tight junction protein claudin-7, new partners, new functions. Front Biosci. 2008;13:5847-65.

45. Ouban A, Ahmed AA. Claudins in human cancer: a review. Histol Histopathol. 2010;25:83-90.

46. Overgaard CE, Daugherty BL, Mitchell LA, Koval M. Claudins: control of barrier function and regulation in response to oxidant stress. Antioxid Redox Signal. 2011;15:1179-93.

47. Jordan NV, Johnson GL, Abell AN. Tracking the intermediate stages of epithelial-mesenchymal transition in epithelial stem cells and cancer. Cell Cycle. 2011;10:2865-73

48. Shaw AR, Li L. Exploration of the functional proteome: lessons from lipid rafts. Curr Opin Mol Ther. 2003;5:294-301.

49. McIntosh TJ. Overview of membrane rafts. Methods Mol Biol. 2007;398:1-7.

50. Epand RM, Thomas A, Brasseur R, Epand RF. Cholesterol interaction with proteins that partition into membrane domains: an overview. Subcell Biochem. 2010;51:253-78.

51. Surma MA, Klose C, Klemm RW, Ejsing CS, Simons K. Generic sorting of raft lipids into secretory vesicles in yeast. Traffic. 2011;12:1139-47.

52. Philip R, Heiler S, Mu W, Büchler MW, Zöller M, Thuma F. Claudin-7 promotes the epithelial-mesenchymal transition in human colorectal cancer. Oncotarget. 2015;6:2046-63.

53. Zheng B, DeRan M, Li X, Liao X, Fukata M, Wu X. 2-Bromopalmitate analogues as activity-based probes to explore palmitoyl acyltransferases. J Am Chem Soc. 2013;135:7082-5.

54. Jordan K, Chodock R, Hand AR, Laird DW. The origin of annular junctions: a mechanism of gap junction internalization. J Cell Sci. 2001;114:763-73.

55. Trebak M, Begg GE, Chong JM, Kanazireva EV, Herlyn D, Speicher DW. Oligomeric state of the colon carcinoma-associated glycoprotein GA733-2 (Ep-CAM/EGP40) and its role in GA733-mediated homotypic cell-cell adhesion. J Biol Chem. 2001;276:2299-309.

56. Balzar M, Winter MJ, de Boer CJ, Litvinov SV. The biology of the 17-1A antigen (Ep-CAM). J Mol Med (Berl). 1999;77:699-712.

57. Auger-Messier M, Turgeon ES, Leduc R, Escher E, Guillemette G. The constitutively active N111G-AT1 receptor for angiotensin II modifies the morphology and cytoskeletal organization of HEK-293 cells. Exp Cell Res. 2005;308:188-95.

58. Runz H, Rietdorf J, Tomic I, de Bernard M, Beyreuther K, Pepperkok R, et al. Inhibition of intracellular cholesterol transport alters presenilin localization and amyloid precursor protein processing in neuronal cells. J Neurosci. 2002;22:1679-89.

59. Tauro BJ, Greening DW, Mathias RA, Mathivanan S, Ji H, Simpson RJ. Two distinct populations of exosomes are released from LIM1863 colon carcinoma cell-derived organoids. Mol Cell Proteomics. 2013;12:587-98.

60. Wu CJ, Mannan P, Lu M, Udey MC. Epithelial cell adhesion molecule (EpCAM) regulates claudin dynamics and tight junctions. J Biol Chem. 2013;288:12253-68.

61. Nübel T, Preobraschenski J, Tuncay H, Weiss T, Kuhn S, Ladwein M, et al. Claudin-7 regulates EpCAM-mediated functions in tumor progression. Mol Cancer Res. 2009;7:285-99.

62. Yamazaki Y, Tokumasu R, Kimura H, Tsukita S. Role of claudin species-specific dynamics in reconstitution and remodeling of the zonula occludens. Mol Biol Cell. 2011;22:1495-504.

63. Piper RC, Katzmann DJ. Biogenesis and function of multivesicular bodies Annu Rev Cell Dev Biol. 2007;23:519-47.

64. Simpson RJ, Lim JW, Moritz RL, Mathivanan S. Exosomes: proteomic insights and diagnostic potential. Expert Rev Proteomics. 2009;6:267-83.

65. Ivanov Al, Nusrat A, Parkos CA. The epithelium in inflammatory bowel disease: potential role of endocytosis of junctional proteins in barrier disruption. Novartis Found Symp. 2004;263:115-24.

66. Ybe JA, Wakeham DE, Brodsky FM, Hwang PK. Molecular structures of proteins involved in vesicle fusion. Traffic. 2000;1:474-9.

67. van der Gun BT, Melchers $\sqcup$, Ruiters MH, de Leij LF, McLaughlin PM, Rots MG. EpCAM in carcinogenesis: the good, the bad or the ugly. Carcinogenesis. 2010;31:1913-21. 
68. Horiuchi K. A brief history of tumor necrosis factor a-converting enzyme: an overview of ectodomain shedding. Keio J Med. 2013;62:29-36.

69. Rose-John S. ADAM17, shedding, TACE as therapeutic targets. Pharmacol Res. 2013;71:19-22.

70. Hansen AG, Arnold SA, Jiang M, Palmer TD, Ketova T, Merkel A, et al. ALCAM/CD166 is a TGF- $\beta$-responsive marker and functional regulator of prostate cancer metastasis to bone. Cancer Res. 2014;74:1404-15.

71. Denzel S, Maetzel D, Mack B, Eggert C, Bärr G, Gires O. Initial activation of EpCAM cleavage via cell-to-cell contact. BMC Cancer. 2009;9:402.

72. Bhat AA, Pope JL, Smith JJ, Ahmad R, Chen X, Washington MK, Beauchamp $R D$, Singh $A B$, Dhawan P. Claudin-7 expression induces mesenchymal to epithelial transformation (MET) to inhibit colon tumorigenesis. Oncogene. 2014 Dec 15. doi: 10.1038/onc.2014.385. [Epub ahead of print]

73. Goldenring JR, Nam KT. Rab25 as a tumour suppressor in colon carcinogenesis. Br J Cancer. 2011;104:33-6.

74. Mitra S, Cheng KW, Mills GB. Rab25 in cancer: a brief update. Biochem Soc Trans. 2012;40:1404-8.

75. Cheng JM, Volk L, Janaki DK, Vyakaranam S, Ran S, Rao KA. Tumor suppressor function of Rab25 in triple-negative breast cancer. Int J Cancer. 2010;126:2799-812.

76. Leth-Larsen R, Terp MG, Christensen AG, Elias D, Kühlwein T, Jensen ON, et al. Functional heterogeneity within the CD44 high human breast cancer stem cell-like compartment reveals a gene signature predictive of distant metastasis. Mol Med. 2012;18:1109-21.

77. Gabison EE, Hoang-Xuan T, Mauviel A, Menashi S. EMMPRIN/CD147, an MMP modulator in cancer, development and tissue repair. Biochimie. 2005;87:361-8.

78. Chen H, Lam Fok K, Jiang X, Chan HC. New insights into germ cell migration and survival/apoptosis in spermatogenesis: Lessons from CD147. Spermatogenesis. 2012;2:264-72.

79. Cannon SC, Strittmatter SM. Functional expression of sodium channel mutations identified in families with periodic paralysis. Neuron. 1993;10:317-26

80. Rana S, Yue S, Stadel D, Zöller M. Toward tailored exosomes: the exosomal tetraspanin web contributes to target cell selection. Int J Biochem Cell Biol. 2012;44:1574-84.

81. Brigidi GS, Bamji SX. Detection of protein palmitoylation in cultured Hippocampal neurons by immunoprecipitation and acyl-biotin exchange (ABE). J Vis Exp. 2013;72, e50031. doi 10.3791/50031.

82. Gobom J, Schuerenberg M, Mueller M, Theiss D, Lehrach H, Nordhoff E. Alpha-cyano-4-hydroxycinnamic acid affinity sample preparation. A protocol for MALDI-MS peptide analysis in proteomics. Anal Chem. 2001;73:434-8.

\section{Submit your next manuscript to BioMed Central and take full advantage of:}

- Convenient online submission

- Thorough peer review

- No space constraints or color figure charges

- Immediate publication on acceptance

- Inclusion in PubMed, CAS, Scopus and Google Scholar

- Research which is freely available for redistribution 